\title{
Development of the Captive Breeding Programme for the Re-introduction of Hazel dormice (Muscardinus avellanarius) in the United Kingdom, 1982-1994
}

\author{
Dot Eaton $^{1, *}$, Martin Hicks ${ }^{2}$ \\ ${ }^{1}$ Berkshire, UK \\ ${ }^{2}$ Hertfordshire Ecology, Environment Department, Hertfordshire County Council, UK
}

Copyright $\bigcirc 2017$ by authors, all rights reserved. Authors agree that this article remains permanently open access under the terms of the Creative Commons Attribution License 4.0 International License

\begin{abstract}
In 1981 the lead author wished to instigate a breeding programme for an indigenous and endangered British mammal species that was known to have suffered a significant decline in numbers. Captive breeding to provide individuals for potential re-introductions was considered the most appropriate approach to helping the re-establishment of wild populations from where they had previously been lost or declined. The selected species needed to be harmless to the environment and small so that expensive techniques were not required. The intention was to generate a sufficiently large and genetically diverse captive population which would supply a formal re-introduction programme into appropriate sites. Having addressed the reasons for previous declines of local extinction, released dormice would provide the basis for new populations. Given the resources available, the Common or Hazel dormouse, Muscardinus avellanarius was considered to be a suitable species for this programme. Successful captive breeding had not been achieved previously. The Mammal Society Dormouse Survey 197579 demonstrated it had been lost from seven counties in the UK during the previous 100 years [1]. A nocturnal and secretive species little was known about the detailed dietary requirements or behaviour of the common dormouse. The establishment and development of the breeding colony enabled observations of their nocturnal activity in captivity. This was observed at very close quarters and behaviour related to feeding, mating, juvenile development and other seasonal changes in activity was noted. Initially there were numerous juvenile deaths within the colony, usually in spring and autumn but with changes to the temperature regime immediately prior to and post hibernation, this mortality reduced. By 1992 the numbers in the captive breeding colony grew to 33 individuals, sufficient for the first trial re-introduction of 11 individuals to take place in Hertfordshire following the IUCN guidelines [4]. Following further breeding and survival success, further re-introductions were supported and by 1995 the colony had risen to 97 individuals. In that year the Common Dormouse Captive Breeders Group was formed and currently six
\end{abstract}

dormouse breeders in the group donate individuals each year to re-introduction projects, supervised by The People's Trust for Endangered Species.

Keywords Common or Hazel dormouse, Muscardinus avellanarius, Captive Breeding

\section{Introduction}

In the United Kingdom there is one indigenous species of dormouse, the Common or Hazel dormouse Muscardinus avellanarius. This is a small, mouse-sized mammal, adults weighing between 15 - 30grammes. It is much smaller than the only other species of dormouse present in the UK, the Fat or Edible dormouse Glis glis, a rat-sized mammal of 50-250g and a recent (1902) introduction. Glis remains largely localised to the Chiltern Hills and although it is spreading it is not considered to represent a threat to the native dormouse. An adult Hazel dormouse has bright golden or orange-brown fur and a semi-prehensile tail. It has long whiskers, round ears and large black eyes set on the side of its head. It is nocturnal and hibernates over winter for up to seven months of the year. In the late $19^{\text {th }}$ Century Hazel dormice were to be found throughout most of the wooded countryside in England and Wales. However, the Hazel dormouse Survey 1975-79 organised by The Mammal Society (Hurrell and Macintosh 1984) [1] found it had disappeared from seven counties in the UK. It is fully protected under the Wildlife and Countryside Act 1981 being included on Schedules 5 and 6. It was hoped that the establishment of a successful captive breeding colony would supply dormice for re-introductions to help address the decline in the species.

\section{Objectives}

Having identified the Hazel dormouse as a suitable species to support with a captive breeding programme, the project 
identified a number of requirements and research aims:

1. To determine on the optimal physical conditions for keeping Hazel dormice in captivity. This would reflect known food and habitat preferences of wild dormice;

2. To acquire an initial stock of Hazel dormice to establish a captive breeding colony;

3. To maintain suitable genetic diversity of the breeding colony;

4. To ensure appropriate husbandry to maintain the health of individuals suitable for breeding;

5. To record health, mortality and loss;

6. To observe breeding and seasonal changes in behavior;

7. To ensure the breeding colony reached sufficient size to enable individuals to be used for a re-introduction;

8. To establish a wider captive-breeding programme to support re-introduction projects nationally.

\section{Project Delivery}

\subsection{To Determine on the Optimal Physical Conditions for Keeping Hazel dormice in Captivity. This Would Reflect Known Food and Habitat Preferences of Wild Dormice}

\section{Habitat Requirements in Captivity}

In the early 1980s there was little information recorded formally on the food preferences of dormice or habitat requirements. However, Hurrell (1980) [2] had noted Hazel dormice could most often be found in shrubby woodland with hazel. Therefore, part of the investigation was to study different stages of re-growth found in a woodland site known to support Hazel dormice. Wallis Wood in Surrey was one of the few remaining managed hazel copses in the south of England, owned by the Surrey Wildlife Trust and coppiced on a ten to twelve-year rotation.

Coppicing is an ancient practice dating back well over 1000 years where areas of trees and shrubs (a 'coupe') are felled to nearly ground level to encourage regeneration of a future harvestable regrowth in the form of many straight stems. These were cut again in anything from three to 25 years depending on the proposed use of the 'underwood' crop, such as for hurdles or firewood. Standard, high-canopy trees were also present (around 10-12 per acre) providing a timber crop, but not sufficient to create extensive shading of the coppice. This management creates significant habitat diversity within woodland including very dense regrowth of between three and around seven years old, which along with ground flora and other vegetation provides an impenetrable shrub layer habitat valued by variety of wildlife including birds such as warblers and nightingales, as well as small mammals such as the Hazel dormouse.

In Wallis Wood the layout of coppice coupes and their ages can be seen in Figure 1. In 1982/3 three ages of coppice were surveyed for plants likely to be used by dormice. These were:
1. A thirty-year-old section of the wood due to be coppiced in $1983 / 84$. This was dark and overshadowed by ash Fraxinus excelsior. There was much bare ground with only moss and young ash seedlings.

2. A four-year-old coppice last coppiced in 1977/8. This coupe supported abundant hazel Corylus avellana two or three metres high. The ground flora was dominated by bramble Rubus fruticosus up to a metre and sanicle Sanicula europaea was also present. Honeysuckle Lonicera periclymenum was present but still at a young stage of growth and mostly at ground level.

3. A coupe which had been recently coppiced in 1979/80. This was covered with young hazel regrowth of $30 \mathrm{~cm}$ and a ground flora consisting of ash saplings, sanicle, clumps of stinging nettle Urtica dioica, rosebay willow herb Chamerion angustifolium and thistle Cirsium $s p$. with quite a lot of bare patches of ground.

The surveys demonstrated the habitat diversity in composition and structure within the wood and in particular the dense vegetation of the younger age coppice. This is consistent with the type of vegetation considered to be important for Hazel dormice and confirmed the need to reflect this in the captive colony in order to support successful breeding.

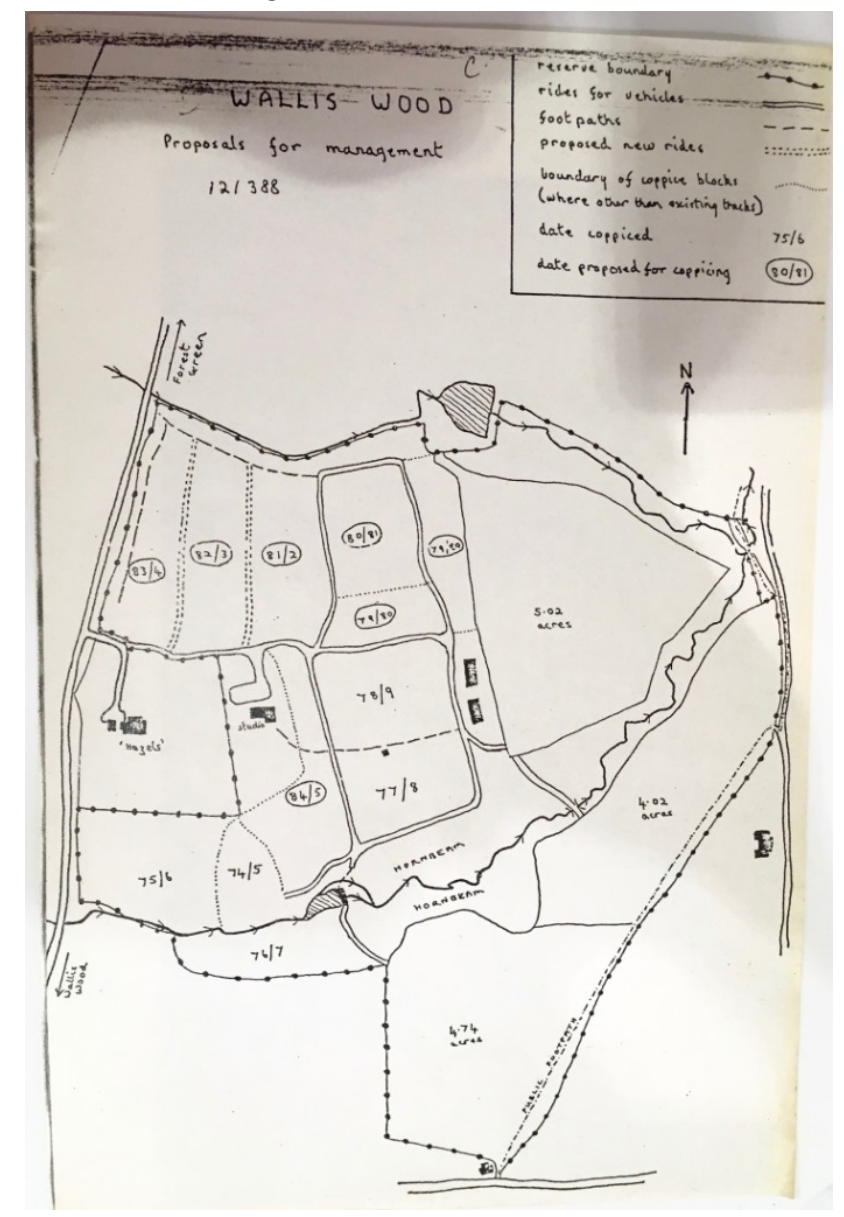

Figure 1. Map of Wallis Wood, Surrey showing layout of coppice compartments and winters when cut. 


\subsection{Development of Breeding Enclosures}

Following this research, in 1982/3 an outdoor enclosure was constructed at Chessington Zoo within a former large animal cage. This was subsequently improved by the construction of a cage measuring four metres by two metres and two metres high. It was planted out with young hazel, bramble and rosebay willow herb. This was attached to a one cubic metre enclosed feeding station, lit with infra-red light. The dormice would occasionally run in to take food or water, which enabled some nocturnal sightings but no natural behavioural activity could be seen. The external cages were not illuminated; therefore the dormice could not easily be seen at night.

Due to a change in circumstances, the breeding colony was moved to Windsor Safari Park in 1988 when the dormice were moved into a shed which was divided into four internal compartments. Quarter inch gauge wire netting was used to divide each compartment which had a door to enable feeding and cleaning. There was a small viewing space in the middle of the shed. Nest boxes were fixed at different heights within each compartment. Each compartment was branched out with fresh branches twice a week. An infra-red light was also fixed inside to the roof of the shed and now it was possible to observe the dormice activity in detail at night.

In 1988, following an article by British naturalist Robin Page in the national Magazine 'Country Life,' funds were made available by the Valerie White Trust. This funding enabled a larger Breeding Unit to be constructed, which enabled better observation of the dormice at night. The breeding unit consisted of two large wooden sheds joined end to end. This was divided into eight compartments, each separated by clear Perspex. Each compartment measured two metres high, 40 centimetres wide and 40 centimetres deep. Each compartment could hold a pair or small group of dormice. The Perspex was to avoid direct contact between individual dormice to limit the potential spread of any infections and also prevent fighting between males. Within each compartment, a nest box was provided for each adult animal and fixed at various heights, from one metre to the top of the compartment. A viewing corridor along the length of the sheds had infra-red lighting which lit the compartments and enabled the dormice to be observed without disturbance potentially at a very close range of a few inches.

Funding also enabled four large external enclosures rising to three metres to be added to four of the internal breeding compartments to provide access externally to more natural habitat and weather acclimatisation for dormice in these compartments (Fig.2).

\subsection{To Acquire an Initial Stock of Hazel dormice to Establish a Captive Breeding Colony}

In 1982 there were no known Hazel dormice in captivity. Following considerable efforts to contact naturalists and other interested parties for information on dormice which could be used as initial breeding stock, contact was made with BBC cameraman Owen Newman who had recently trapped and filmed a dormouse giving birth. Subsequently a license from the Nature Conservancy Council was obtained by D. Eaton to trap and take wild dormice with O. Newman for the purposes of establishing captive breeding group. In 1983 three dormice were trapped in Wiltshire using Longworth small mammal traps to provide the first individuals of the captive colony.

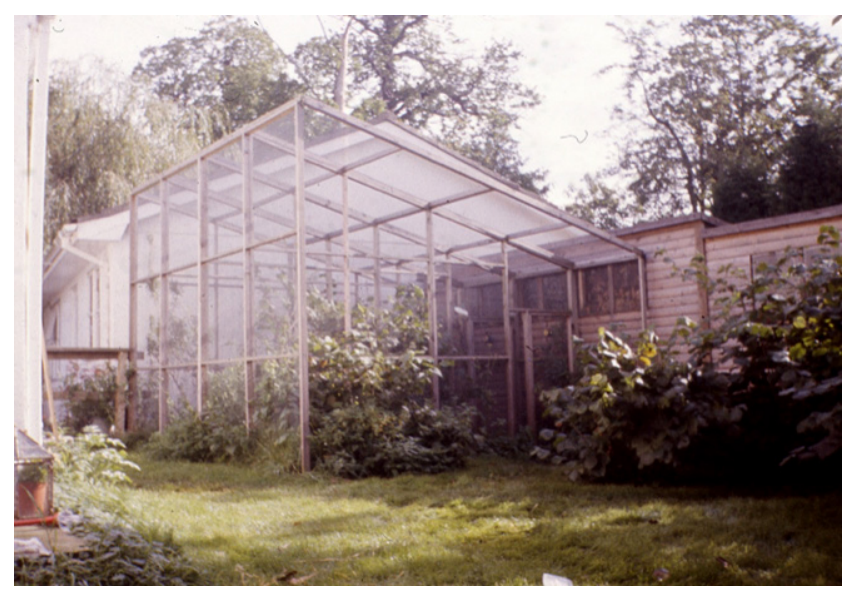

Figure 2. Captive Breeding Unit at Windsor Safari Park

\subsection{To Maintain Suitable Genetic Diversity of the Breeding Colony}

The three original individuals were from the same woodland site in Wiltshire, so it was important to widen the genetic variety by introducing further individuals from elsewhere. Initially this was difficult as there were no other captive breeders but subsequently this became easier as other captive colonies were formed such as at Cheddar in Somerset (Doug Woods) in 1986 and at London Zoo in 1989.

In 1985 three more wild dormice were added to the original three dormice from elsewhere in Wiltshire, as well as a juvenile female found by a member of the public in the New Forest in Hampshire. This had been kept in captivity over winter until the following summer.

In 1989, two adult dormice were exchanged with individuals from London Zoo and added to the breeding stock. 1990 two further adults were donated, but not mixed. Unfortunately, these individuals died.

In 1993, the colony was supplemented by two adult wild Hazel dormice, obtained under licence, held by Andrea McKenna of the Zoological Society of London (ZSL).

The sex of the above individuals was determined when they were added to other dormice in each enclosure to ensure there was a mix of males and females.

\subsection{To Ensue Appropriate Husbandry to Maintain the Health of Individuals Suitable for Breeding}

Wild growing food as fresh browse was provided twice a week for the captive dormice according to the published references to their diet (Hurrell [2] and Richards [3]) and the Wallis Wood study, when it was available and in season. 
This included flowers, fruits, seeds and browse from a range of species such as hawthorn, blackberry, apple blossom, honeysuckle, beech, hornbeam, hazel and sweet chestnut.

Supplementary food was also offered daily throughout the summer months in the form of a dried food mix of sunflower seeds, peanuts, puppy meal, "Vitaline" (a high protein dry dog food), "Sluice" (an insect based bird food) as well as fruit including apple, pear, grape and cherries. Water was also provided.

The observed dormice showed preferences for feeding alone and not sharing food in a group, so it was necessary to offer many opportunities for individuals to feed separately. Berries and apple sections were placed on cut branches at different locations within the cages. It was also necessary to offer each individual dormouse a nest box, so that sufficient numbers of nest boxes were available to enable the opportunity for solitary nesting in order to avoid creating any further stressful conditions. However, during hibernation up to five dormice were known to share a nest box.

To keep the dormice free from external parasites they were powdered with Louse powder before waking from hibernating and re-treated again a week later. It was decided to powder before the animals were fully active to prevent them grooming excessively and ingesting the louse powder. Also as many external parasites lay eggs in the fur of the host animal which hatch in about a week, a second treatment of powder was considered necessary. The dormice were treated again at the end of the autumn season, by which time, the majority of the animals had become torpid.

The bedding in the nest boxes was also changed before and after hibernation. The bedding consisted of dried meadow grass, shredded paper or straw. Agricultural baled hay was not used as this can go musty and potentially cause the dormice to develop breathing problems.

In autumn, two thirds of the nest boxes were placed at ground level and insulated using garden mulch in order to reduce temperature fluctuations during hibernation. Most of the dormice chose to hibernate in the lower, ground level nest boxes.

\subsection{To Record Health, Mortality and Loss}

From 1987, Dr. John Lewis, of International Zoo Veterinary Practice, provided ongoing veterinary support. In general, the captive dormice exhibited good health with no signs of behavioural or physical distress. However, where they showed limited signs of illness these included:

These were seen very occasionally and considered to be stress or parasite related.

Precautionary treatment for external parasites was undertaken as outlined above.

The potential for stress was avoided by:

a) Offering a large number of feeding stations;

b) Providing enough nest boxes to enable solitary use;

c) Construction of separate indoor enclosures to avoid fighting between males; d) Keeping compatible pairing or groups of individuals together;

e) Avoiding disturbance or handling of dormice, particularly during the months when they are most active - May to October.

\section{Adult Dormouse Deaths}

These were recorded as follows:

October 1885 Two individuals;

October 1987 One individual;

1989 Two individuals;

1990 Two individuals (not mixed with other dormice);

October 1992 Seven individuals - from eating bad peanuts;

1993 One individual.

In a small number of cases where post mortems were undertaken, the cause of death was determined to be: a split thoracic duct, gallstones, an extended bladder.

\section{Juvenile Deaths}

Mortality of juveniles became an ongoing problem as soon as the colony began to breed successfully. The juveniles were not sent for post mortem, but were weighed in a number of cases.

October 1985 Six individuals, each $8 \mathrm{~g}$ in weight;

May 1986 Eleven individuals;

October 1987 Two individuals, each $10 \mathrm{~g}$;

1989 Ten individuals, each $15 \mathrm{~g}$;

1990 Nine individuals died;

October 1992 Three individuals, each 12g;

1993 Two individuals.

\section{Other Reasons for Loss}

1990 Four individuals disappeared.

1992 A small hole had been dug into the outside enclosure, following which seven dormice had disappeared. It was considered this was due to predation by a weasel.

1993 Three partially eaten juvenile dormouse bodies were found.

\subsection{To Observe Breeding and Seasonal Changes in Behaviour}

\section{3-1987}

During this period, when dormice were active at night it was possible to see their silhouettes moving within the external cages. Although they were breeding, unfortunately it was not possible to observe in any great detail their nocturnal activity as the outside cages were not illuminated.

\section{8-1994}

Following the installation of the infra-red lights in the corridor which illuminated the eight inside breeding compartments, the dormice were observed in the breeding unit for different lengths of time, two or three nights a week from April to November. Each of the eight compartments 
held male and female dormice, either as a pair or two females to one male.

\subsection{Results of Observations}

\section{Feeding Behaviour}

Of the natural foods taken, dormice were seen to take buds and the small part of the base of a leaf, particularly beech and hazel. They were seen to spend a lot of time on oak browse, not taking the leaves themselves but probably the associated insects. They were observed to eat a variety of flowers in the spring including hawthorn, wild rose, blackberry, apple blossom and willow herb. The insect-based "Sluice" was also eaten throughout the summer. In autumn, beech nuts, hornbeam seeds, hazel nuts and sweet chestnuts were taken. Apple is taken throughout most of the active season if made available.

Dormice appear to feed alone throughout the summer. When one dormouse is feeding and another dormouse approaches the food source, such as a piece of apple or the dry food mix on the feeding shelf, only the dominant individual remains whilst the other subordinate dormouse runs away. However, in the autumn prior to hibernation when fattening up, two or more individuals were seen feeding together. At such times, increased levels of food are taken.

Whilst eating a flower such as rosebay willow herb, a dormouse was observed balancing by holding onto the stem with its back paws and curling its semi-prehensile tail round the stem for balance, whilst holding the flower with its front paws whilst the flower is eaten. When eating a nut such as a hazel nut, the dormouse was seen holding the nut in its front paws while sitting upright and turning the nut round as its opens the shell to reach the kernel. This leaves a very smooth, circular hole enabling dormouse-opened hazels nuts to be identified in the field. Once a hazel nut has ripened and hardened it is harder for the dormice to open and soft green nuts are preferred.

In mid- to late-August the adult dormice begin to feed excessively in order to gaining weight prior to entering hibernation in October. The juveniles continued to be active after this for several more weeks whilst they also gained weight prior to hibernation.

Whilst not tested during this study, it was considered that the onset of additional feeding prior to hibernation may also have been stimulated by the length of daylight as well as later temperature changes, given that August temperatures remain high.

\section{Nest Building}

Throughout the captive breeding period 1983 - 1994, although the building of a dormouse nest was not observed, on several occasions two or three leaves were seen joined together. These were considered to be practice nests.

The gathering of nesting material was seen. On one occasion, a dormouse was observed stripping the bark from a honeysuckle branch; a small piece of bark was held in the teeth, the front paws pushed against the branch while the animal's head was jerked back several times as the bark, which is soft and pliable was stripped off. The section of honeysuckle bark was then carried, (some in the mouth and some dragging behind,) up into the nest box. Another time, a beech leaf was observed being bitten off at the base, where the leaf joins the branch. This took several seconds, after which the whole leaf was then crammed into the mouth of the dormouse and carried into a nest box.

\section{Seasonal and Other Behaviour}

During hibernation, the rate of breathing is seen to be one or two breaths a minute. On waking the dormice were found to slowly uncurl and breathing becomes gradually more rapid. Once fully awake, they become very active and could be observed running, grooming themselves and feeding in the branches of the enclosures or on the feeding station.

When startled, dormice were observed to stay motionless wherever they are, either crouching behind a leaf or just staying still. This 'frozen' posture lasts for about half a minute before they run quickly away or jump to escape or carry on with their pre- startled activity.

\section{Observation of Breeding Behaviour}

Within a week or ten days after waking from hibernation usually mid to late May - male dormice were observed chasing females frantically and squeaking for about three consecutive nights. This was considered to be pre-mating behaviour as once the female had been mated, the chasing ceased and the animals settled down to their normal activity of feeding, self-grooming and exploring the plants. In some enclosures, the three-night chasing would start again ten nights later. This was found to occur if the female had not become pregnant, and had therefore come into oestrus again.

In the enclosures where the female had been mated, the young were born thirty days after conception. This was noticed when a female did not emerge from the nest box for one or sometimes two nights.

Due to the design of the breeding unit, the viewing corridor was very close to the enclosures and if a female ran or stayed still on the wire of the enclosure, it was possible to see whether she was suckling young.

Juvenile dormice emerged from the nest box about thirty days after they were born. The first few nights after leaving the next box, the inexperienced juveniles displayed greater naive behaviour, such as not staying still when perceived danger threatened or jumping or running too soon. During the first few nights out of the nest box, the female and occasionally the male dormouse were observed guiding the young.

Breeding can also occur late in the season to produce a second litter, mostly in August.

\section{Impact of Temperature Changes}

On three separate occasions, twice in October 1985 and 
once in October 1987, litters of dormice died in the nest although they were fully furred and very nearly ready to leave the nest. In each case the female was fat and torpid and the night-time temperature had dropped for several nights. Numerous underweight juveniles died in the months of May and October, when temperatures fluctuate most.

However, in autumn 1991 from late September to late October, the infra-red lights were left on overnight continuously for four weeks and the temperature did not fall below 10 degrees C. Juvenile deaths reduced significantly and no more lactating females died before their young were able to leave the nest. This represented a significant breakthrough in reducing mortality within the breeding colony.

\subsection{To Ensure the Breeding Colony Reached Sufficient Size to Enable Individuals to Be Used for a Re-introduction}

Although it had been known for some time that hazel dormice could easily be kept in captivity (8), there were no records of any that had been bred in captivity.

Although the dormice had started to breed from the start of the breeding colony in 1983, the numbers of individuals that were lost to mortality effectively mirrored the births, so in the early years the overall numbers were not increasing as had been hoped. The dormice were counted in May of each year. Numbers of individuals rose from the original three dormice in 1983 to five in 1985, to nineteen in 1986. However, after the deaths of eleven juveniles, the May count in 1987 was five dormice, followed by ten individuals in May 1988. After successful breeding seasons, the numbers of dormice rose to 22 individuals in both 1989 and 1990 , although there were losses of 12 and 15 individuals in both years respectively. In the May of 1991 there were 20 dormice, but there remained an equal number of losses. Given the ongoing mortality levels, this population was still considered too low to support a re-introduction.
However, following the overnight use of the infra-red lights in the autumn of 1991, the spring count in 1992 was 33 individuals consisting of 21 adult dormice and 12 successfully over-wintered juveniles from the previous year. Given this breakthrough it was now considered possible for the breeding colony to support a release into the wild.

In 1992, a trial re-introduction of dormice took place in Hertfordshire. Eleven individuals were used from the breeding unit, consisting of two females and one male with two juveniles and a second family of one female with five juveniles.

This re-introduction was undertaken at the instigation of Julian Ford-Robertson (Senior Science Master at Haileybury College, Hertford) supervising 16 Advanced Level students, with support from Martin Hicks, Ecologist at Hertfordshire Environmental Records Centre, Steve Whitbread, dormouse ecologist at Southampton University, Professor John Gurnell, Queen Mary University of London and D. Eaton.

The Haileybury re-introduction was carried out following the IUCN guidelines and was reported in Re-introduction News [4], and will be described in the proceedings of the 10th International Dormouse Conference held in 2014 [ in press].

Having discovered the importance of temperature control prior to and also immediately following hibernation, the colony now began to increase in size as dormouse mortality declined in subsequent years.

In spring 1993 the adult numbers in the colony had reached 30. Breeding was successful in this year enabling six individuals to be used to reinforce the re-introduction at Haileybury.

By May 1994 the numbers of dormice had risen to 83 individuals enabling 20 to be provided for a re-introduction at a new site supported by English Nature. By spring 1995 the colony, now based at Burnham Beeches had risen to 97 dormice (Fig. 3). 


\section{Number of adult dormice in breeding colony each year - includes numbers that died and numbers that were introduced}

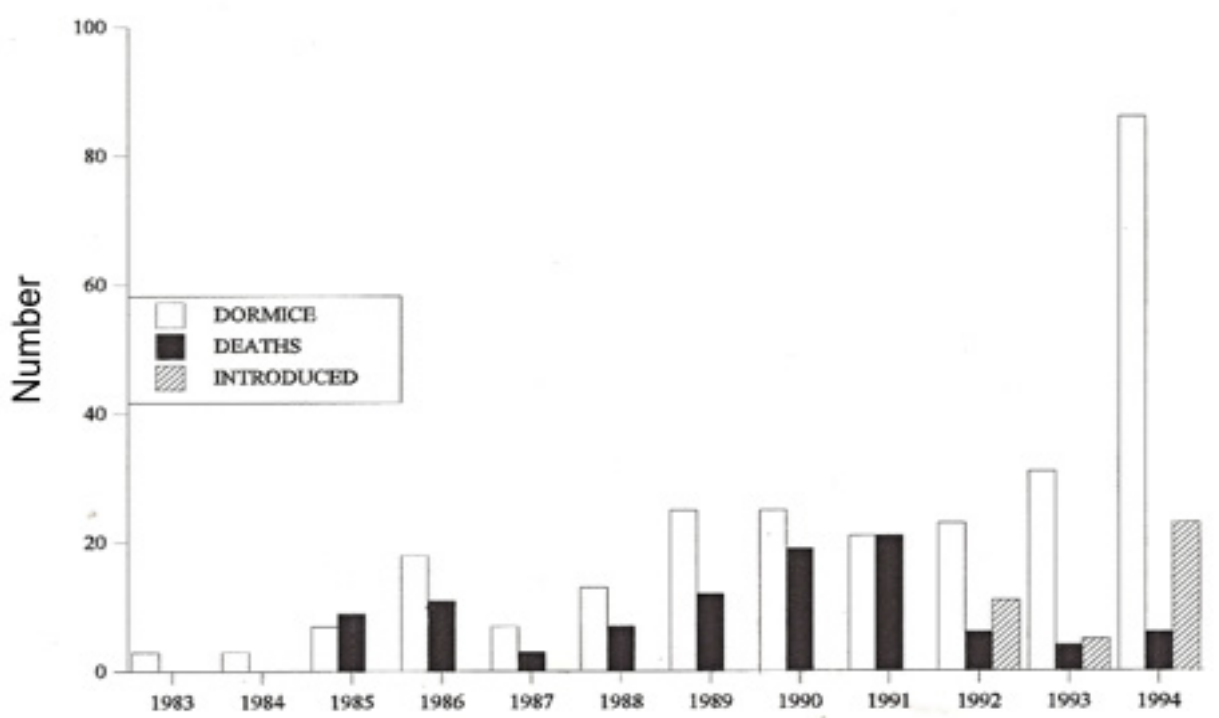

Figure 3. Graph shows numbers of adult dormice in the breeding colony in May each year. Graph also shows deaths and re-introduced animals

\subsection{To Establish a Wider Captive Breeding Programme to Support re-introduction Projects Nationally}

In October 1992, two months after the Haileybury re-introduction, Windsor Safari Park went into receivership and the Breeding Centre had to be moved.

A potential location was found at Burnham Beeches, a large ancient woodland complex, north-west of London in Buckinghamshire, owned by the Corporation of London. The move to Burnham was completed by early September 1994. On 14th September 1994 a contract was drawn up between Dot Eaton and Burnham Beeches, giving joint ownership of the Captive Breeding Unit to the Cooperation of London and D. Eaton. It was also suggested that a meeting should be organised to consider a more formal approach to dormouse breeding and re-introduction programme nationally.

In February 1995 a meeting was held at the Mammal Society in London. Those attending included Dot Eaton, captive dormouse breeder; Dr. Pat Morris and Dr. Paul Bright, Royal Holloway College, London; Dr. Tony Mitchell-Jones, Mammal specialist, English Nature; Doug Woods, captive dormouse breeder; Dr. John Lewis, International Veterinary Group; Dr. Helen Reed, Ecologist at Burnham Beeches and Martin Hicks, Ecologist, Hertfordshire Environmental Records Centre. It was suggested that a group of dormice breeders should be formed and this is now known as the Common [Hazel] Dormouse Captive Breeders Group, CDCBG.

By 2017 the CDCBG had established six captive breeding units around the UK, run by a range of private breeders, Zoos and Wildlife Parks. Neil Bemment, Curator of Mammals at
Paignton Zoo is Chairman. A stud book is maintained by Judi Dunn of the Wildwood Centre. All members meet in April each year and discuss matters related to:

- numbers of dormice in each captive breeders centre;

- Information on pre-released veterinary checks from the previous year;

- how many individual dormice each breeder can give to the following year's release;

- where the following year's release is planned and how many dormice are needed.

Each breeder brings to the meeting their dormice for release that year, the numbers of which were agreed the previous year. Half of these animals go to Tony Sainsbury, Veterinary Surgeon at London Zoo, or Ghislaine Sayers Veterinary surgeon at Paignton Zoo. The dormice are given a thorough health check for six or seven weeks, before going to the release site.

The People's Trust for Endangered Species (PTES) now, have a dedicated Dormouse Officer (Ian White) who co-ordinates the releases. The PTES have now organised a total of 26 re-introductions into 14 counties in the UK. The dormice for these re-introductions are supplied by the members of the Common Dormouse Captive Breeders Group.

In addition to this the National Dormouse Monitoring Programme (NDMP) was set up by Dr P Morris in 1983 to monitor the numbers of Hazel dormice in the wild. This continues to be perhaps the largest terrestrial mammal monitoring programme in the UK and in 2016 over 400 sites were monitored using nest boxes by licensed volunteers, 
recording over 6,000 individuals.

The Forestry Commission (Ben Ditchburn) is gathering further evidence by means of the National Forest Inventory using aerial photo maps of all British woodlands and ground observations to provide details of the structure and condition of the habitat resource. It is planned to use this evidence to assess the suitability of woodlands for hazel dormice [9].

Current research at Exeter University (Cecily Goodwin), supported by PTES, the Forestry Commission and the Woodland Trust is also investigating how "habitat loss, fragmentation and habitat quality determines dormouse numbers and how this might interact with climate change." [10]

\section{Conclusions}

1. The first successful captive breeding colony of the Hazel dormouse, an endangered native British mammal, was established by Dot Eaton at Chessington Zoo and then Windsor Safari Park between 1983 and 1994. It was moved again to Burnham Beeches after which the Common [Hazel] Dormouse Captive Breeders Group was established. This group now supplies dormice as part of a formal re-introduction programme to supplement wild populations, organised by the People's Trust for Endangered Species.

2. It was necessary to observe the behaviour of captive dormice to help inform the best methods of husbandry. The final design of the captive breeding unit with external and internal cages and infra-red lamps enabled observations of dormice including breeding behaviour and mating, social interaction, seasonal changes such as increased feeding and fattening pre-hibernation. This was achieved without causing any disturbance to the animals to reduce any additional stress.

3. Juvenile dormice were most at risk when air temperature dropped below 10 degrees $\mathrm{C}$. for several consecutive nights. The success of the breeding programme changed markedly when continued use of infra-red lamps prevented such a drop. This is likely to be one of the factors that influence the long-term survival of wild populations of dormice. More research is required to demonstrate this conclusively but this species does appear to be very sensitive to temperature fluctuations, particularly early and late in the active season. Consequently, the changing temperature regimes as a result of climate change could be a major factor in influencing the conservation of the dormouse.

4. Food preferences in the captive colony were observed to change through the season. Consequently, it is reasonable to assume that a wide diversity of plant species and associated insects is needed to provide a sequence of flowering and fruiting vegetation and associated invertebrate prey necessary to support populations in the wild. This assertion is supported by Bright and Morris [5]. In 2016 PTES emphasised the importance of encouraging appropriate woodland management to provide optimum habitat conditions and create habitat corridors between fragmented sites [7].

5. Although the captive breeding and reintroduction programme has been successful, the National Dormouse Monitoring Programme has recorded a 38\% decline in Britain in Hazel dormouse numbers over the last ten years.

6. Loss of suitable habitat as a result of the decline in traditional woodland management remains critical for a species which requires such a specialised habitat and range of foods. It is a major factor in the dramatic decline of the dormouse, in addition to the potential impacts of climate change and increased habitat fragmentation due to build development pressure. These issues need addressing to ensure that the requirements of the Hazel dormouse are maintained and enhanced. This is supported by ongoing monitoring and research into their biology and conservation, augmented by the work of the captive breeding programme pioneered in the early 1980 s, as reported here.

\section{REFERENCES}

[1] The Mammal Society Dormouse Survey 1975 - 79 published 1984

[2] The Hazel Dormouse, E. Hurrell Blandford Press 1980

[3] The Food of The Hazel Dormouse (Muscardinus avellanarius) in South Devon Mammal Review. 1984 C. Richards, A. White, E. Hurrell, E. Price

[4] Re-introduction News No 6 May 1993 D. Eaton, M. Hicks, S. Whitbread, J. Ford-Robertson

[5] Why is Dormice Rare? A Case Study in Conservation Biology Mammal Review 1996. Volume 26 No 4 P. Bright, P. Morris

[6] A New Survey of the Dormouse Muscardinus avellanarius in Britain 1993-4 - The Great Nut Hunt. Mammal Review 1996 Volume 26 No 4 P. Bright, P. Morris, A. Mitchell-Jones

[7] Dormouse Decline is worse than Expected. Ian White PTES Slough Observer 16th September 2016

[8] Dormouse chapter, The Handbook of British Mammals, 1960. H.N. Southern, with H. G. and E. Hurrell

[9] Investigating hazel dormouse population trends and the causes of declines. Cecily Goodwin. National Dormouse Conference 2016 (PTES) Presentation/Proceedings

[10] The National Forest Inventory. Ben Ditchburn. National Dormouse Conference 2016 (PTES) Presentation /Proceedings. 